\title{
Vitamin D and Diabetes Mellitus Type 2
}

Ifigenia Kostoglou-Athanassiou, Panagiotis Athanassiou, Anastasios Gkountouvas, Philippos Kaldrymides

Department of Endocrinology, Red Cross Hospital, Athens, Greece Department of Rheumatology, St. Paul's Hospital, Thessaloniki, Greece

Department of Endocrinology, Metaxa Hospital, Pireaus, Greece 


\section{Introduction}

- Vitamin D deficiency has been observed in diabetes mellitus type 2 patients

- It has been found to be related to poor glycemic control in diabetes mellitus type 2 patients as well as in patients with gestational diabetes

- The administration of vitamin D in diabetes mellitus type 2 patients with vitamin D deficiency has been found to have conflicting results on blood glucose control 


\section{Aim}

- The aim was to assess the effect of vitamin $D$ administration in diabetes mellitus type 2 patients with vitamin $D$ deficiency on blood glucose control 


\section{Methods}

- In a group of 20 diabetes mellitus type 2 patients with vitamin D deficiency vitamin D was administered along with oral hypoglycemic agents

- 25(OH) $D_{3}$ and glycosylated hemoglobin levels were measured at the beginning of the study and 3 months later

- Patients were on treatment with oral hypoglycemic agents

- Cholecalciferol was administered orally at a dose of 1200 iu daily for a period of 3 months 


\section{Results}

- At the beginning of the study diabetes mellitus type 2 patients were found to have vitamin D deficiency, 25(OH) $D_{3}$ levels being $18.6 \pm 0.86$ $\mathrm{ng} / \mathrm{ml}$ (mean \pm SEM), glycosylated hemoglobin levels being $7.1 \pm 0.15 \%$

- After the administration of cholecalciferol for a period of 3 months glycosylated hemoglobin levels decreased to $6.56 \pm 0.19 \%(p<0.05$, Student's t test) 


\section{Conclusions}

- Vitamin D supplementation in diabetes mellitus type 2 patients on oral hypoglycemic agents may contribute to better blood glucose control

- These results are in accordance with the known effect of vitamin D on insulin secretion as well as on insulin sensitivity

- However, as the study involved diabetes mellitus type 2 patients the effect of better adherence to dietary restrictions or improved compliance to the oral hypoglycemic treatment on blood glucose control cannot be excluded 